\title{
Early Morning Neuroparalytic Syndrome
}

Sir,

A 14-yr-old girl presented with abnormal sensations all over her body when she got up from sleep early in the morning. She had no other significant past medical history. On examination, her vitals were stable with a normal systemic examination. Her blood investigations, chest X-ray and electrocardiogram were normal. She subsequently developed an acute paralysis with respiratory failure that required intubation and continuous mechanical ventilation. At this point, she had partial ptosis, dilated pupils, complete external ophthalmoplegia and flaccid quadriparesis with a flexor plantar response. A differential diagnosis of hypokalemic paralysis, myasthenic crisis, drug overdosage, Miller Fischer syndrome and early morning neuroparalytic syndrome (EMNS) were considered. Her cerebrospinal fluid examination and electroencephalogram were normal. With a working diagnosis of EMNS, she was started on anti-snake venom (ASV) and atropine-neostigmine. Within 3 hours, she began to show a spontaneous respiratory effort; though she required mechanical ventilation for another 12 hours. This recovery with ASV suggests the possibility of an elapid envenomation. The ptosis and ophthalmoplegia resolved over the next two days and she was discharged after four days with no focal neurological deficits.

EMNS is a rare presentation of the elapid bite that is commonly seen among farmers and slum dwellers that sleep out in the open environment. ${ }^{1,2}$ These patients are brought to the hospital with a history of ptosis and paralysis with no bite marks or local skin changes on the body. This is characteristic of the nocturnally active kraits whose bites are generally painless with minimal to no skin changes. Kularatna et al. ${ }^{3}$ found that $60-70 \%$ of snakebites occurred while the patients were asleep with $17 \%$ of these having undetectable bite marks on the body.
Elapid neurotoxicosis acts at the peripheral neuromuscular junctions either post-synaptically or pre-synaptically causing paralysis. ${ }^{4,5}$ This paralysis is first detected as bilateral ptosis and external ophthalmoplegia, gradually progressing to involve the muscles of deglutition. This generalized flaccid paralysis may occur with consciousness provided the patient is not in circulatory failure. The pre- paralytic symptoms include paresthesia and numbness. The neurotoxic effects spontaneously resolve over several days or in response to antivenom and anticholinesterase.

In conclusion, a differential diagnosis for an acute onset of flaccid paralysis should include EMNS in snakebite endemic areas and ASV should be started immediately even in the absence of snakebite marks.

\author{
Mohammed Haneef ${ }^{1}$, Donae E. George and \\ Abraham S Babu \\ CSI Mission Hospital \\ ${ }^{1}$ Consultant Physician, Koyili Hospital, Kannur-670004, \\ Kerala, India \\ E-mail:mhaneef@gmail.com
}

\section{REFERENCES}

1. Sain R. Snake poisoning presenting as early morning neuroparalytic syndrome in Jugghi dwellers. JAPI 1986; 34:415-417.

2. Srimannarayana J, Dutta TK, Sahai A, Badrinath S. Rational Use of Anti-snake Venom (ASV): Trial of Various Regimens in Hemotoxic Snake Envenomation. JAPI 2004; 52:789-793.

3. Kularatna SAM. Common krait in Anuradhapura, Sri Lanka: a prospective clinical study,1996-98. Postgraduate Medical Journal 2002;78:276-280.

4. Juckett G, Hancox JG. Venomous snake bites in United States: Management, review and update. Am Fam Physician 2002; 65:1367-1374

5. Dutta TK, Muktha V. Snake bite. JIMA 2006;104:250-254. 